\title{
Verruca Vulgaris Arising Within the Red Portion of a Multicolored Tattoo
}

\author{
Dev Sahni, MD, MHA; Laurel Wong Cummings, MD; Rachel Balow Lee, MD; Joi Lenczowski, MD; \\ Mark Cameron Mochel, MD
}

\section{PRACTICE POINTS}

- Various adverse reactions and infectious agents may involve tattoos.

- Verruca vulgaris may affect tattoos in a colorrestricted manner and demonstrate latency of many years after tattoo placement.

- Timely diagnosis of the tattoo-involving process, confirmed by biopsy, allows for appropriate management. reported no prior trauma to the area, prior rash or irritation, or similar changes to her other tattoos, including those with red ink. The affected tattoo was inscribed at a separate time from the other tattoos. Physical examination of the irritated tattoo revealed hyperkeratotic papules with firm scaling in the zone of dermal red pigment (Figure 1). Notable nodularity or deep induration was not present. The clinical differential diagnosis included a hypersensitivity reaction to red tattoo ink, sarcoidosis, and an infectious process, such as an atypical mycobacterial

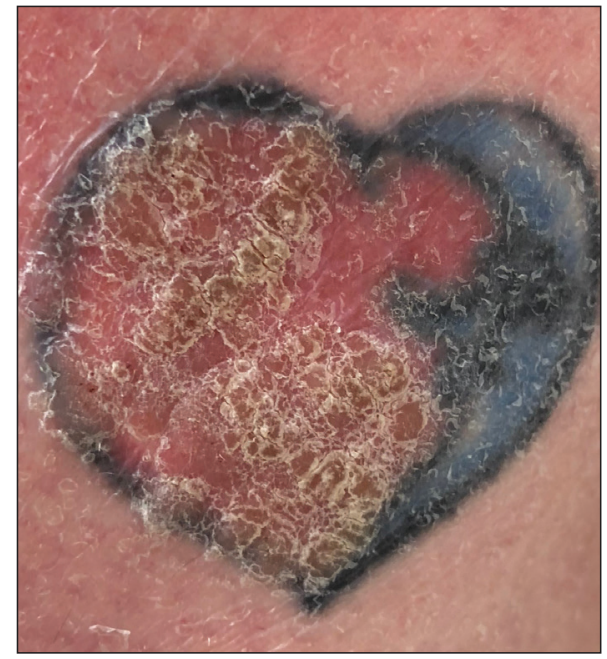

FIGURE 1. Scaly papules coalescing into small plaques, largely confined to the red-inked area of a tattoo with only focal involvement of the black-inked rim.
To the Editor:

The art of tattooing continues to gain popularity in the 21st century, albeit with accompanying hazards. ${ }^{1}$ Reported adverse reactions to tattoos include infections, tumors, and hypersensitivity and granulomatous reactions. ${ }^{2}$ Various infectious agents may involve tattoos, including human papillomavirus (HPV), molluscum contagiosum, herpes simplex virus, hepatitis $C$ virus, tuberculoid and nontuberculoid mycobacteria, and Staphylococcus aureus. ${ }^{2}$ Verruca vulgaris infrequently has been reported to develop in tattoos. ${ }^{3,4}$ Previously reported cases of verruca in tattoos suggest a predilection for blue or black pigment. ${ }^{1-5}$ We report a case of verruca vulgaris occurring within the red-inked areas of a tattoo that first appeared approximately 18 years after the initial tattoo placement.

A 44-year-old woman presented with erythema, induration, and irritation of a tattoo on the left leg of 2 years duration. The tattoo initially was inscribed more than 20 years prior. The patient had a history of type 2 diabetes mellitus and chronic obstructive pulmonary disease. She

Dr. Sahni is from the Department of Dermatology, University of Utah Health, Salt Lake City. Dr. Cummings is from Commonwealth Dermatology, Richmond, Virginia. Drs. Lee, Lenczowski, and Mochel are from the Department of Dermatology, Virginia Commonwealth University, Richmond. Dr. Mochel also is from the Department of Pathology.

The authors report no conflict of interest.

Correspondence: Mark Cameron Mochel, MD, Departments of Pathology and Dermatology, Virginia Commonwealth University Health System, 1200 E Marshall St, Gateway 6, Richmond, VA 23298 (Mark.Mochel@VCUHealth.org).

doi:10.12788/cutis.0350 
infection. A punch biopsy demonstrated papillomatous epidermal hyperplasia with hyperkeratosis, focal parakeratosis, and frequent vacuolization of keratinocytes with enlarged keratohyalin granules, diagnostic of verruca vulgaris (Figure 2). Of note, the patient did not have clinically apparent viral warts elsewhere on physical examination. The patient was successfully managed with a combination of 2 treatments of intralesional
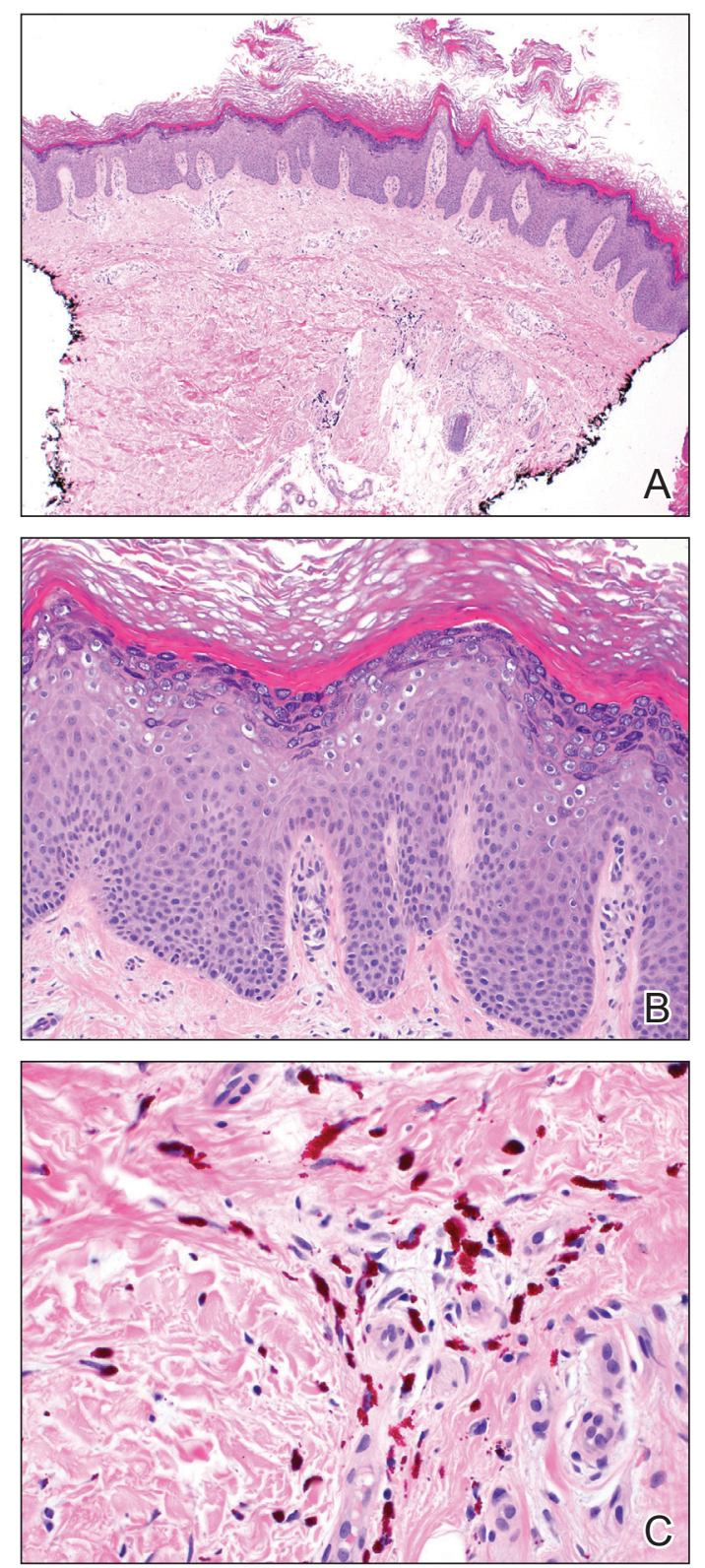

FIGURE 2. Histopathologic findings of verruca vulgaris. A, Verrucous epidermal changes and dermal pigment $(\mathrm{H} \& \mathrm{E}$, original magnification $\times 40$ ). B, Epidermal acanthosis and papillomatosis with viral cytopathic changes $(H \& E$, original magnification $\times 200)$. C, Underlying dermal red tattoo pigment $(\mathrm{H \& E}$, original magnification $\times 400)$.
Candida antigen and 3 treatments of cryotherapy with resolution of most lesions over the course of 8 months. Over the following several months, the patient applied topical salicylic acid, which led to the resolution of the remaining lesions. The verrucae had not recurred 19 months after the initial presentation.

The development of verruca vulgaris within a tattoo may occur secondary to various mechanisms of HPV inoculation, including introduction of the virus through contaminated ink, the tattoo artist's saliva, autoinoculation, or koebnerization of a pre-existing verruca vulgaris. ${ }^{4}$ Local immune system dysregulation secondary to tattoo ink also has been proposed as a mechanism for HPV infection in this setting. ${ }^{1,5}$ The contents of darker tattoo pigments may promote formation of reactive oxygen species inducing local immunocompromise. ${ }^{5}$

The pathogenic mechanism was elusive in our patient. Although the localization of verruca vulgaris to the zones of red pigment may be merely coincidental, this phenomenon raised suspicion for direct inoculation via contaminated red ink. The patient's other red inkcontaining tattoos that were inscribed separately were spared, compatible with contamination of the red ink used for the affected tattoo. However, the delayed onset of nearly 2 decades was exceptional, given the shorter previously reported latencies ranging from months to 10 years. ${ }^{4}$ Autoinoculation or koebnerization is plausible, though greater involvement of nonred pigments would be expected as well as a briefer latency. Finally, the possibility of local immune dysregulation seemed feasible, given the slow evolution of the lesions largely restricted to one pigment type.

We report a case of verruca vulgaris within the red area of a multicolored tattoo that occurred approximately 18 years after tattoo placement. This case highlights a rare presentation of an infectious agent that may complicate tattoos. Both predilection for red pigment rather than black or blue pigment and the long latency period raised interesting questions regarding pathogenesis. Confirmatory biopsy enables effective management of this tattoo complication.

\section{REFERENCES}

1. Huynh TN, Jackson JD, Brodell RT. Tattoo and vaccination sites: possible nest for opportunistic infections, tumors, and dysimmune reactions. Clin Dermatol. 2014;32:678-684.

2. Wenzel SM, Rittmann I, Landthaler M, et al. Adverse reactions after tattooing: review of the literature and comparison to results of a survey. Dermatology. 2013;226:138-147.

3. Trefzer U, Schmollack K, Stockfleth E, et al. Verrucae in a multicolored decorative tattoo. J Am Acad Dermatol. 2004;50:478-479.

4. Wanat KA, Tyring S, Rady P, et al. Human papillomavirus type 27 associated with multiple verruca within a tattoo: report of a case and review of the literature. Int J Dermatol. 2014;53:882-884.

5. Ramey K, Ibrahim J, Brodell RT. Verruca localization predominately in black tattoo ink: a retrospective case series. J Eur Acad Dermatol Venereol. 2016;30:E34-E36. 